\title{
Lower bounds on scintillation detector timing performance *
}

\author{
Neal H. Clinthorne ${ }^{1)}$, Alfred O. Hero III ${ }^{2)}$, Nicholas A. Petrick ${ }^{2)}$, and W. Leslie Rogers ${ }^{1)}$ \\ ${ }^{1)}$ Division of Nuclear Medicine and ${ }^{2)}$ Department of Electrical Engineering and Computer Science, University of Michigan, \\ Ann Arbor, MI 48109-0552, USA
}

Fundamental method-independent limits on the timing performance of scintillation detectors are useful for identıfying regimes in which either present timing methods are nearly optimal or where a considerable performance gain might be realized using better pulse processing technıques. Several types of lower bounds on mean-squared tıming error (MSE) performance have been developed and applied to scintillation detectors. The simple Cramèr-Rao (CR) bound can be useful in determining the limiting MSE for scintillators having a relatıvely high rate of photon production such as $\mathrm{BaF}_{2}$ and $\mathrm{NaI}(\mathrm{Tl})$; however, it tends to overestımate the achievable performance for scintillators with lower rates such as BGO. For this reason, alternative bounds have been developed using rate-distortion theory $[1,2]$ or by assuming that the conversion of energy to scintillation light must pass through excited states which have exponential lifetime densities [3]. The bounds are functions of the mean scintillation pulse shape, the scintillation intensity, and photodetector characteristics; they are simple to evaluate and can be used to conveniently assess the limiting timing performance of scintillation detectors.

\section{Introduction and background}

Accurate determination of $\gamma$-ray interaction time in a scintillation detector is necessary in many areas of radiation measurement. Our motivation stems from positron emission tomography (PET) where the timeseries of interaction time estimates from a ring of detectors is used to determine whether or not a pair of detected $\gamma$-rays originated from the same electronpositron annihilation. To improve PET image accuracy, we have been investigating improved timing methods for scintillation detectors by applying statistical estimation theory, but it is natural to ask: What is the margin for possible improvement over present timing methods? Lower bounds on the mean-squared timing error which are independent of the timing method are useful for exactly this purpose and can provide a yardstick against which the performance of current timing techniques can be measured. In addition these bounds can be useful in assessing performance sensitivity to scintillator and photomultiplier parameters and they can indicate the weak link in the timing chain.

In this paper, we present several lower bounds on the mean-squared timing error (MSE) derived from a stochastic model for the scintillation detector we have developed. The purpose of this paper is to bring together several expressions we have developed and illustrate their use. We have omitted detailed derivations

\footnotetext{
* This work was supported by the National Cancer Institute, DHHS, under PHS grant CA46622.
}

and refer the interested reader to previous work [1-3]. In the next section we present the model for the scintillation detector from which the bounds were derived. Section 3 presents expressions for the lower bounds which are evaluated in section 4 .

\section{Scintillation detector model}

A valid mathematical model is a key element for the development of useful lower bounds and improved timing methods. A combination Poisson point-process model and additive Gaussian noise model has been developed to describe the timing performance of scintillation detectors.

We assume that the process of scintillation photon emission can be characterized by an inhomogeneous Poisson point-process in which the random sequence of $N_{\text {T }}$ photoelectron arrival times at the first dynode of a photomultiplier can be completely described by its mean arrival rate, defined as:

$\lambda(t-\tau)=\eta \int_{0}^{t} f(s) \lambda_{\mathrm{u}}(t-s-\tau) \mathrm{d} s+\lambda_{\mathrm{d}}$

The mean rate or intensity $\lambda(t-\tau)$ in eq. (1) is essentially the scintillator response, $\lambda_{\mathrm{u}}(t)$, to a $\gamma$-ray interaction at time, $\tau$, smoothed by additional time dispersions, $f(s)$, due to phototube transit-time jitter or optical collection time dispersion. The factor $\eta$ represents the counting efficiency of the photodetector and $\eta_{\mathrm{d}}$ is the dark pulse rate due to either extraneous photons 
emitted from the scintillator or electrons from the photocathode.

The model we have employed to describe the photomultiplication or avalanche process has been adopted from optical communications and represents the output current of the photodetector as a superposition of randomly scaled impulse responses driven by the Poisson point-process described above. The random output current $X(t)$ of the photodetector due to a photoelectron arrival time sequence $\{t\}_{t=1}^{N_{t}}$, is given by

$X(t)=\sum_{t=1}^{N} a_{t} p\left(t-t_{i}\right)+W(t)$

where $p(t)$ is the energy-normalized single-electron time response (SER) of the photodetector, $a_{1}$ is the random photodetector gain sequence, assumed to be independent, ıdentically-dıstributed, and drawn from a known probability density (pdf), and $W(t)$ is an additive white Gaussian electronic noise of power level $N_{0} / 2 \mathrm{~Hz}^{-1}$. For this model, assuming a causal photodetector impulse response $p(t)$, the likelihood function for observing the output waveform given the $\gamma$-ray interaction time can be written:

$$
\begin{aligned}
f(X(t) \mid \tau)= & E\left[\operatorname { e x p } \left(-\int_{0}^{T}(X(t)\right.\right. \\
& \left.\left.\left.-\sum_{t=1}^{\nu_{\mathrm{T}}} a_{t} p\left(t-t_{t}\right)\right)^{2} \mathrm{~d} t / N_{0}\right)\right],
\end{aligned}
$$

where the waveform is observed on the interval $[0, T]$ and the expectation is taken over all sequences of arrival times $\left\{t_{t}\right\}$ and all random gain sequences $\left\{a_{t}\right\}$. We assume that the scintillation pulse is fully supported on the observation interval $[0, T]$ and we define the integrated rate or mean number of photoelectrons in this interval as $\Lambda$.

We have performed preliminary validation studies by comparing results from a $\mathrm{BGO} / \mathrm{CsF}$ time-of-flight system with those predicted by Monte Carlo simulations and have found that BGO timing performance can be accurately predicted using this model. More extensive validation studies are currently under way.

\section{Lower bounds on timing performance}

In principle, the limiting MSE for the above model can be evaluated by first deriving the minimum MSE time-of-arrival estimator and then evaluating the corresponding MSE expression; however, since the likelihood function (3) is not in an analytic form this problem is generally intractable and we must resort to approximate MSE expressions. In what follows, we present two distinct classes of lower bounds on the mean-squared timing error. The first assumes that the random arrival time sequence of photoelectrons can be directly observed in the photodetector. These bounds include timing degradations that occur before the multiplication process but cannot account for the effects of multiplication statistics, SER shape, and Gaussian electronic noise. However, for many inorganic scintillators used in conjunction with photomultipliers, the major performance degradations have occurred by this point and the additional statistical effects can be ignored. The second class of bounds we present are based on observations of the photodetector output waveform and can potentially be better performance indicators when the additive Gaussian noise component is large; for example, when an avalanche photodiode is used in place of a photomultiplier.

\section{Direct observatıon bounds}

The global Cramèr-Rao (CR) bound [4] for the problem of estimating $\gamma$-ray interaction time given the direct observations of $\left\{t_{t}\right\}_{l=1}^{N_{1}}$ takes the following form,

$\mathrm{MSE} \geqslant\left[\int_{0}^{T} \frac{\dot{\lambda}^{2}(t)}{\lambda(t)} \mathrm{d} t+E\left(\left(\frac{\partial \ln f_{\tau}(t)}{\mathrm{d} t}\right)\right)^{2}\right]^{-1}$.

Here $f_{\tau}(t)$ is the proor pdf or our prior knowledge of the $\gamma$-ray interaction time and $\dot{\lambda}(t)$ is the time-derivative of the intensity (1). We note that this bound requires that both the intensity and prior density be differentiable. Often, even if these conditions are met, the bound can yield an overly optımistic MSE estimate - especially if the intensity changes abruptly where its magnitude is small. As an example, for the common biexponential representation for the scintillation response, the $\mathrm{CR}$ bound cannot be evaluated because the derivative is unbounded at $t=0$.

To overcome the limitations of the CR bound we developed a new bound in ref. [3] based on the assumption that for scintillators, the lifetime of excited states whose decay leads to the emission of scintillation photons has an exponential pdf. Given direct observation of the photoelectron arrival time sequence and a broad prior density, this bound is exact minimum MSE for a monoexponentially decaying pulse having no dark current. Since the response of many scintillators suggests that there can be several decay constants, the bound has been extended to describe this phenomenon. In particular, for $m$ decay constants, the bound takes the form:

$\mathrm{MSE} \geqslant E_{N_{1}} \quad N_{m}\left[\left(\sum_{t=1}^{m} \frac{N_{t}}{\tau_{t}}\right)^{-2}\right]$,

where $\tau_{t}$ is the $t$ th decay constant and the expectation $1 \mathrm{~S}$ taken over the Poisson distributed random variables $N_{t}$ with means $\alpha_{1} \Lambda$, where $\alpha_{1}$ is the fraction of states 
having mean lifetime $\tau_{t}$ and $\sum_{t=1}^{m} \alpha_{t}=1$. We have found this exponential decay bound to be tight when evaluating scintillators which have a combination of moderate total light output and a relatively long decay such as BGO. It has the disadvantage that it does not describe timing degradations due to the smoothing effects of transit-time jitter or scintillation photon collection and of course, the direct observation bounds in general cannot account for photodetector multiplication statistics and the additive Gaussian electronic noise.

\subsection{Waveform observation bounds}

The second class of bounds we have developed are based on observations of the photodetector output current $X(t)$ and can potentially describe the additional timing degradations due to a non-ideal photodetector. The bound is a composite developed from rate-distortion theory; detailed derivations can be found in refs. $[1,2]$.

The bound is based on the following fundamental inequality from information theory [5],

MSE $\geqslant \frac{1}{2 \pi \mathrm{e}} \mathrm{e}^{2 H(\tau)} \mathrm{e}^{-2 C}$.

$H(\tau)$ is the entropy of the prior pdf of $\gamma$-ray arrival time defined as

$H(\tau)=\int_{-\infty}^{\infty} f_{\tau}(\sigma) \ln f_{\tau}(\sigma) \mathrm{d} \sigma$

and $\mathrm{C}$ is the channel capacity, describing the maximum rate of information transfer from the $\gamma$-ray interaction time to the observation $X(t)$ under the most favorable prior pdf.

We can conceptualize the scintillation detector as a cascade of two communications channels: the first, $c_{1}$, takes the $\gamma$-ray interaction time into the point-process $\left\{t_{t}\right\}_{t=1}^{\mathrm{N}_{1}}$ and the second, $c_{2}$, is an additive Gaussian noise channel, mapping the point-process to the continuous photodetector output. Since, by the dataprocessing theorem, the capacity of the cascade channel can be no greater than the capacity of either channel, we can replace $C$ in eq. (6) by $\min \left\{C_{1}, C_{2}\right\}$, where $C_{1}$ and $C_{2}$ are the capacities of channels $c_{1}$ and $c_{2}$ respectively.

For channel $c_{1}$, we have the following bound on the channel capacity [1]:

$C_{1} \leqslant \int_{0}^{T} \lambda(t) \ln \frac{T \lambda(t)}{\Lambda} \mathrm{d} t$
For the Gaussian noise channel, $c_{2}$, we have the bound [2]:

$$
\begin{aligned}
C_{2} \leq & \frac{-1}{4 \pi} \int_{-\infty}^{\infty} \ln (1 \\
& \left.+\gamma \frac{|P(\omega)|^{2}\left[\Lambda+|F[\lambda(t)-\bar{\lambda}(t)]|^{2}\right]}{\int_{0}^{T} p^{2}(t) \mathrm{d} t}\right) \mathrm{d} \omega,
\end{aligned}
$$

where $P(\omega)$ is the Fourier transform of the photodetector SER, $p(t), F[\cdot]$ represents the Fourier transform operation, and $\bar{\lambda}(t)$ is the intensity function smoothed by the prior pdf, $f_{\tau}(\tau)$. The constant $\gamma$ in (9) is the SER signal-to-noise ratio (SNR) given by,

$\gamma=\frac{2 \bar{a}^{2} \int_{0}^{T} p^{2}(t) \mathrm{d} t}{N_{0}}$,

where $\bar{a}$ is the mean photodetector gain.

Substituting the bounding expressions for $C_{1}$ and $C_{2}$ into (6) yields two lower bounds on the MSE. At low signal-to-noise ratios, the additive Gaussian noise is the dominant degradation while at high SNR the Poisson noise dominates. These bounds have the advantage that they include photodetector effects and are accurate when the rate of scintillation photon emission is extremely low, but unfortunately, they can be very weak when applied at the high signal-to-noise ratios encountered with photomultipliers.

Finally, under the assumptions of a high-rate of scintillation photon emission (such as with $\mathrm{NaI}(\mathrm{Tl})$ ) and a low SNR, an approximate CR bound can be derived by replacing the random signal $\sum_{i=1}^{N_{t}} a_{t} p\left(t-t_{t}\right)$ by its mean, which is merely the intensity function (1) convolved with the photodetector SER. This approximation is justified using the well-known high-intensity Gaussian approximation to shot noise processes [6] and a form of the CR bound [4] for a weak Gaussian signal buried in independent white Gaussian noise. The bound for arrival time estimation is easily derived as:

$\mathrm{CR} 2 \approx\left[\gamma \frac{\int_{0}^{T} \dot{m}^{2}(t) \mathrm{d} t}{\int_{0}^{T} p^{2}(t) \mathrm{d} t}+E\left[\left(\frac{\partial \ln f_{\tau}(t)}{\partial t}\right)^{2}\right]\right]^{-1}$,

where $\gamma$ is again the SNR, $m(t)$ is the mean of $\sum_{i=1}^{N_{t}} a, p\left(t-t_{t}\right)$

$m(t)=\bar{a} \int_{0}^{t} p(s) \lambda(t-s) \mathrm{d} s$,

and $\dot{m}(t)$ denotes the time-derivative of $m(t)$. 


\section{Applications}

We have evaluated the bounds using scintillator parameters typical for $\mathrm{BGO}$ at $511 \mathrm{keV}$ and measured photomultiplier parameters. Parameters for the BGO response were taken from ref. [7]. The response was assumed to be a triexponentral shape having a $2.8 \mathrm{~ns}$ rise time along with $60 \mathrm{~ns}$ and $300 \mathrm{~ns}$ decay times in the ratio of 1:9. The mean number of photoelectrons produced per scintillation was 200 (assuming photoelectric absorption of a $511 \mathrm{keV} \gamma$-ray). The prior uncertainty in the interaction time of the $\gamma$-ray was taken as a broad Gaussian with a standard deviation of $290 \mathrm{~ns}$. The single photoelectron time response was measured from a Burle 8850 photomultıplier.

Fig. 1 plots the direct observation CR bound (CR1) and the exponential decay bound (EDB) against the mean number of photoelectrons in a scintillation. The base-10 log of the mean-squared error in $\mathrm{ns}^{2}$ is plotted as a function of the log of the number of photoelectrons. The CR bound is plotted for several values of an assumed Gaussian photomultiplier transit-time jitter; however, the exponential decay bound is independent of this jitter. We see that at low photoelectron rates the exponentral decay bound is dominant while at higher rates the converse is true. The CR bound shows that the effects of transit-time jitter can be significant even at the moderate photon emission rates for BGO.

For the following studies, a BGO scintillation pulse with $\Lambda=200$ photoelectrons was used. This was convolved with an additional 500 ps FWHM Gaussian pulse to simulate the effects of transit-time jitter.

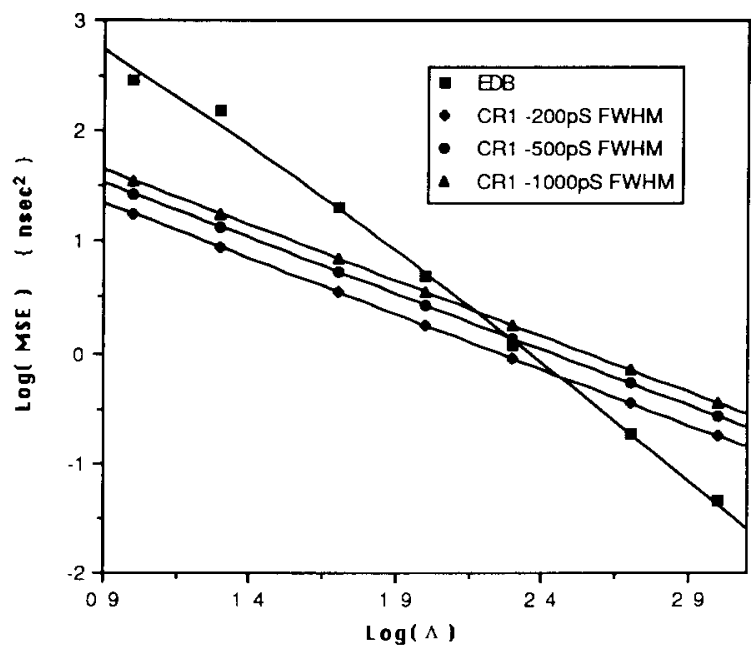

Fig. 1. Lower bounds on mean-square timıng error plotted against the mean number of photoelectrons $(A)$ in a scintillation. The direct observation Cramèr-Rao bounds (CR1) are plotted for photomultıpliers having a Gaussian transit-tıme jitter distribution of 200,500 , and 1000 ps FWHM.

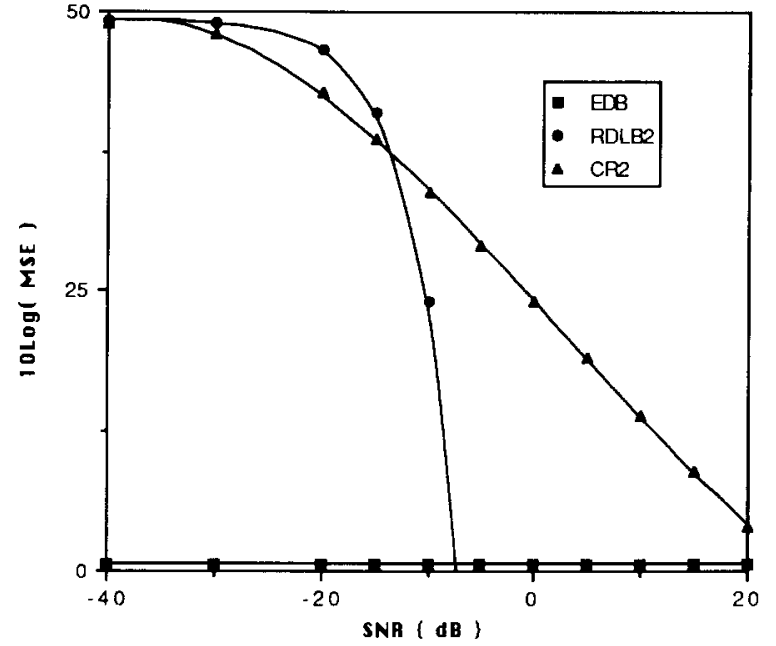

Fig. 2. The Gaussian rate distortion bound (RDLB2) and the high-rate Cramèr-Rao approxımation (CR2) plotted agaınst the photodetector SER signal-to-noise ratio in $\mathrm{dB}$. The exponential decay bound (EDB) for the scintillation pulse has been plotted for reference. Scintillation pulse and phototube parameters are described in the text.

In fig. 2 the effects of an additive white Gaussian noise are shown where the Gaussian rate-distortion bound (RDLB2) and the high-rate CR approximation (CR2) are plotted against the SER signal-to-noise ratio in $\mathrm{dB}$. This SNR is generally worse than would be seen using a photomultiplier but should encompass the situation in which an avalanche photodiode is used instead

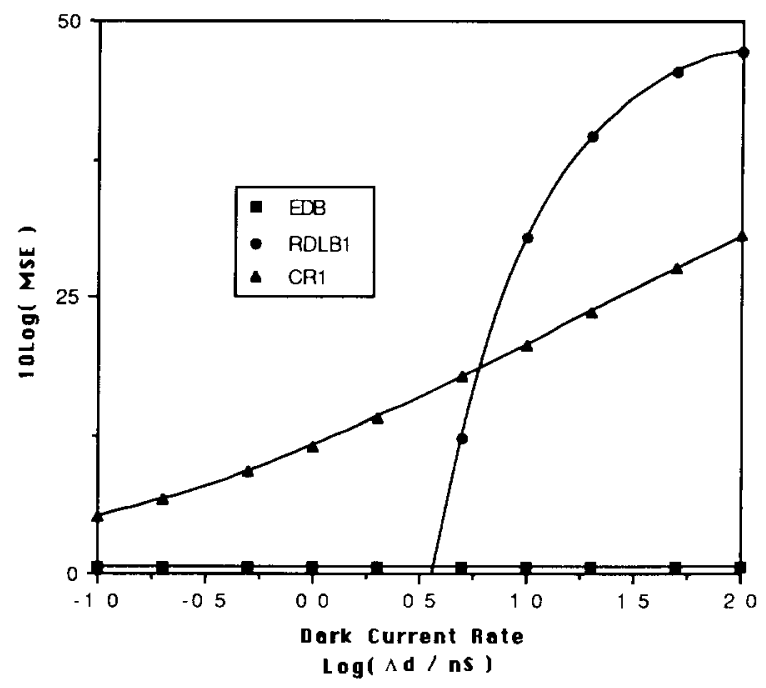

FIg. 3. The Poisson rate-distortion bound (RDLB1) and the direct observation CR bound (CR1) for a BGO scintillation with $A=200$ photoelectrons plotted against the rate of dark pulses The exponential decay bound (EDB), which is not a function of dark current is also plotted. 
of a photomultiplier. At very low SNR, the RDLB2 dominates the $\mathrm{CR}$ bound. At more moderate SNR, however, RDLB2 is extremely weak. The exponential decay bound for $A=200$ is plotted for comparison. It should be recalled, however, that the approximate bound (CR2) is only reliable for low SNR.

Fig. 3 shows results of evaluating the Poisson limited rate-distortion bound (RDLB1) and the direct observation CR bound (CR1) as a function of the background or dark current rate. For low rates, CR1 is the best performance indicator, while at extremely high dark current rates, RDLB1 can be better.

\section{Discussion}

It is apparent that each of the bounds is useful for some range of scintillator and photodetector parameters. For PET, with inorganic scintillators and photomultipliers, the Cramèr-Rao type bounds seem to be the best indicators of the minimum attainable MSE performance. The rate-distortion bounds are very weak in this application except at the very low information limit. When the Cramèr-Rao bounds cannot be evaluated, the exponential decay bound may be a good Indication of the achievable performance - especially if the rate of scintillation photon emission is low-to-moderate.

Accurate evaluation of the expressions presented here requires accurate measurement of photodetector and scintillator parameters - especially for the CR bounds where time-derivative information of the scintillation response is required. A more accurate characterization of the additive Gaussian noise is also necessary. For convenience, we assumed the Gaussian noise distribu- tion to be white; however, both the rate-distortion bound and the CR approximation can easily account for nonwhite, wide-sense stationary noise by replacing $N_{0}$ with the noise power spectrum $N(\omega)$ in the frequency domain formulations. This should be useful in modeling the effects of $1 / f$ noise on timing.

\section{Conclusion}

Lower bounds are useful in evaluating whether or not present timing methods can be improved upon and in indicating the sensitivity of timing performance to various scintillator and photodetector parameters. We have presented several lower bounds on the minimum attainable MSE and briefly illustrated their application. To make this work accessible we are currently rewriting and extensively documenting the lower bound evaluating programs is ANSI C. The source files are expected to be available from the authors from approximately July 1, 1990.

\section{References}

[1] A.O. Hero, Proc. 20th Conf. on Information and Systems Science, Princeton, NJ, 1988, p. 140.

[2] A.O. Hero, IEEE Trans. Info. Theory, IT-35 (4) (1989) 843.

[3] N.H. Clinthorne, N.A. Petrick, W.L. Rogers and A.O. Hero, IEEE Trans. Nucl. Sci. (1990) in press.

[4] H.L. Van Trees, Detection, Estimation, and Modulation Theory: Part I (Wiley, New York, 1968).

[5] R.E. Blahut, Principles and Practice of Information Theory (Addison-Wesley, Reading, MA, 1987).

[6] D.L. Snyder, Random Point Processes (Wiley, New York, NY, 1975).

[7] M. Moszynskı, C. Gresset, J. Vacher and R. Odru, Nucl. Instr. and Meth. 188 (1981) 403. 